\title{
Promoção da Saúde: adesão municipal às ações de combate ao sobrepeso e obesidade infanto-juvenil
}

\author{
Health Promotion: municipal membership to actions to fight child overweight \\ and obesity
}

\section{AUTORES \\ Silvia Veridiana Zamparoni Victorino ${ }^{1}$ (D) \\ Bianca Machado Cruz Shibukawa ${ }^{1}$ (D) \\ Gabrieli Patricio Rissi ${ }^{1}$ (D) \\ Ieda Harumi Higarashi ${ }^{1}$ id \\ 1 Universidade Estadual de Maringá, Maringá, Paraná, Brasil.}

\section{CONTATO}

Bianca Machado Cruz Shibukawa

bih.cruuz@gmail.com

Avenida Itororó, n. 1388, Maringá, Paraná,

Brasil. CEP: 87010-460.

DOI

$10.12820 /$ rbafs. $24 \mathrm{e} 0102$

\begin{abstract}
RESUMO
Objetivou-se analisar a adesão municipal às ações de promoção da saúde relacionadas às atividades físicas e práticas corporais, para crianças e adolescentes para o enfrentamento do sobrepeso e obesidade. Estudo exploratório, transversal, quantitativo. Os sujeitos da pesquisa corresponderam aos $30 \mathrm{mu}-$ nicípios pertencentes à 15a Regional de Saúde do Estado do Paraná. Coletaram-se os dados através de um questionário estruturado aplicado aos gestores informantes de cada município e consulta aos relatórios públicos do Sistema de Vigilância Alimentar e Nutricional, no período de abril a junho de 2014. Analisou-se os dados por estatística descritiva simples e matriz de vizinhança com a co-ocorrência de ação entre os municípios, sendo utilizada como suporte para construção e análise de rede. Verificou-se que, em 2008, as crianças e adolescentes apresentavam 31\% ( $\mathrm{n}=37.588)$ de sobrepeso e $24 \%(\mathrm{n}=19.635)$ de obesidade, sendo um percentual com crescimento gradativo alcançando $40 \%(\mathrm{n}=$ 46.878) e 43\% ( $n=36.997)$ em 2013, respectivamente. A quantidade de ações de promoção em saúde implementadas nos municípios e relacionadas à atividade física variou de $35 \%$ a $80 \%$, dos quais $56,7 \%$ desenvolviam entre $35 \%$ a $50 \% ; 26,7 \%$ de 51 a $65 \%$ e $16,6 \%$ desenvolviam ações entre 66 a $80 \%$ das preconizadas pelo Ministério da Saúde. Nenhum município realiza mais de $80 \%$ das ações preconizadas, o que reforça a necessidade da atuação interdisciplinar no enfrentamento do sobrepeso/obesidade infanto-juvenil, reconhecendo os obstáculos estruturais, humanos e logísticos para a busca de soluções que conduzam um estilo de vida mais saudável, com vistas à maior qualidade de vida futura.
\end{abstract}

Palavras-chave: Promoção da saúde; Obesidade pediátrica; Atividade física; Saúde da criança.

\section{ABSTRACT}

The objective of this study was to analyze municipal adherence to health promotion actions related to physical activities and body practices, for children and adolescents to face overweight and obesity. Exploratory, cross-sectional, quantitative study. The research subjects corresponded to the 30 municipalities belonging to the 15th Regional Health Region of the State of Parana. The data were collected through a structured questionnaire applied to the informing managers of each municipality and consulted the public reports of the Food and Nutrition Surveillance System, from April to June 2014. Data were analyzed by simple descriptive statistics and neighborhood matrix with co-occurrence of action among municipalities, being used as support for network construction and analysis. In 2008, children and adolescents presented 31\% $(n=37,588)$ of overweight and $24 \%(n=19,635)$ of obesity, a percentage with gradual growth, reaching $40 \%(n=46,878)$ and $43 \%(n=36,997)$ in 2013, respectively. The number of health promotion actions implemented in the municipalities and related to physical activity varied from $35 \%$ to $80 \%$, of which $56.7 \%$ developed between 35\% and 50\%; 26.7\% from 51 to 65\% and 16.6\% developed actions between 66 and $80 \%$ of those recommended by the Ministry of Health. No municipality performs more than $80 \%$ of the recommended actions, which reinforces the need for interdisciplinary action to face overweight / obesity in children and adolescents, recognizing the structural, human and logistical obstacles to the search for solutions that lead to a healthier lifestyle, with a view to greater future quality of life.

Keywords: Health promotion; Pedriatric obesity; Physical activity; Child health.

\section{Introdução}

A obesidade é uma doença que se manifesta por meio do armazenamento de gordura no organismo, o que pode ocasionar complicações metabólicas. Pode ser definida ainda como uma doença decorrente de um processo desequilibrado entre gasto e suprimento ener- gético, resultando em ganho de peso, podendo se manifestar nos indivíduos de qualquer faixa etária, etnia ou situação sócio econômica ${ }^{1,2}$.

A população mundial está enfrentando uma epidemia de sobrepeso (SP) e obesidade (OB), fato este expressado pela triplicação de população obesa entre 
1975 a 2016. Estima-se que cerca de 381 milhões de crianças e adolescentes de 0 a 19 anos estejam com sobrepeso ou obesidade ${ }^{3}$.

No Brasil, seguindo-se a tendência apontada por outros países, também é possível observar as taxas de $\mathrm{SP}$ e $\mathrm{OB}$ crescendo exponencialmente, sendo o sexo feminino o mais afetado. Tal aumento sugere ainda a insuficiência ou ineficiência de políticas públicas voltadas para este agravo ${ }^{4}$.

No Paraná, as taxas de SP e OB infantil aumentaram em até $400 \%$ na última década. A temática ganhou destaque nos planos diretores do Estado, incentivando medidas de enfrentamento juntamente com a população, já que na região do estudo $38,14 \%$ das crianças e adolescentes apresentaram $\mathrm{SP}$ e/ou $\mathrm{OB}^{5}$.

Outro fator que contribui com o aumento do SP e $\mathrm{OB}$ na população vigente consiste no próprio processo de urbanização aliado ao estilo de vida adotado. As brincadeiras tornam-se cada vez mais tecnológicas, restringindo ou anulando as possibilidades de movimento, além da modificação dietética onde os alimentos ultraprocessados estão cada vez mais presentes no cotidiano familiar, o que contribui com o desenvolvimento de uma dieta obesogênica podendo implicar em riscos à saúde ${ }^{6}$.

Desta forma, a OB é reconhecida como um grave problema de saúde pública e, diante do cenário epidemiológico do grupo de doenças crônicas não transmissíveis (DCNT), a mesma se destaca por ser simultaneamente uma doença e um fator de risco modificável ${ }^{7}$. Portanto, como medida de enfrentamento a este agravo, o Ministério da Saúde (MS) lançou, em 2011, o Plano de Ações Estratégicas para o Enfrentamento das Doenças Crônicas Não Transmissíveis (PAEDCNT) no Brasil, objetivando a promoção, o desenvolvimento e a implementação de políticas públicas efetivas e integradas, visando reduzir a prevalência de $\mathrm{OB}$. Nesse sentido, a ênfase do plano desenvolvido pelo MS incluiu as atividades físicas, alimentação saudável, combate ao tabagismo e uso de álcool ${ }^{6,8}$.

A Política Nacional de Promoção da Saúde (PNPS) ${ }^{9}$ prevê que a alimentação saudável, associada à prática de atividade física, constitui-se como o fundamento das ações de promoção da saúde (PS), além de ser uma medida estratégica no enfrentamento das deficiências nutricionais, do $\mathrm{SB}$ e da $\mathrm{OB}^{10}$.

A PNPS se articula com os ideais do Sistema Único de Saúde (SUS) e se configura como um novo paradigma, contrapondo-se às práticas tradicionais do modelo biomédico, articulando e reforçando distintas iniciati- vas promocionais e em diferentes níveis de complexidade, encontrando maior espaço na Atenção Básica de Saúde (ABS) e na Estratégia Saúde da Família (ESF). Sabe-se que as ações de promoção à saúde dentro da Atenção Primária em Saúde (APS) voltadas à estimulação de práticas alimentares saudáveis e atividades físicas são essenciais na redução da obesidade e dos gastos com a saúde pública. Entretanto, frequentemente as mesmas se encontram permeadas por desafios, muitos dos quais envolvem a gestão no âmbito da articulação intersetorial ${ }^{11}$.

Neste contexto, objetivou-se analisar a adesão municipal às ações de OS relacionadas às atividades físicas e práticas corporais para crianças e adolescentes, para o enfrentamento do SB e OB.

\section{Métodos}

Este artigo foi extraído da dissertação "Ações para o enfrentamento da obesidade e sobrepeso em crianças e adolescentes nos municípios da 15a Regional de Saúde do Estado do Paraná", apresentada ao Programa de Pós-graduação em Enfermagem da Universidade Estadual de Maringá, em 2014.

O estudo respeitou os aspectos éticos contidos nas Resoluções 466/2012 ${ }^{12}$ e 510/2016 ${ }^{13}$ do Conselho Nacional de Saúde. Aprovou-se o projeto pelo Comitê Permanente de Ética em Pesquisa envolvendo Seres Humanos da Universidade Estadual de Maringá, conforme parecer 623.548/2014.

Trata-se de um estudo exploratório, transversal e de abordagem quantitativa sobre as ações de promoção da saúde voltadas ao público infanto-juvenil na atenção básica em saúde, realizado no âmbito da $15^{\mathrm{a}}$ Regional de Saúde do Estado do Paraná, a qual engloba 30 municípios da região noroeste do Estado apresentando um total de 724.215 habitantes ${ }^{14}$.

Inicialmente, submeteu-se a pesquisa à apreciação pela direção da $15^{\text {a }}$ Regional de Saúde do Estado do Paraná, obtendo-se a adesão de todos os municípios. Os sujeitos da pesquisa corresponderam aos 30 municípios pertencentes à $15^{\text {a }}$ Regional de Saúde do Estado do Paraná e os informantes corresponderam aos Gestores Municipais de Saúde ou algum profissional designado pelo mesmo que possui conhecimento das ações de PS desenvolvidas no âmbito municipal.

Realizou-se uma reunião prévia com cada gestor municipal, visando viabilizar as visitas aos municípios. Coletaram-se os dados por meio de um questionário estruturado aplicado com os gestores municipais e con- 
sulta aos relatórios públicos do Sistema de Vigilância Alimentar e Nutricional (SISVAN). Investigou-se as características sociodemográficas dos gestores municipais, assim como o perfil das ações de PS de cada unidade diante das Secretarias de Saúde e Educação além dos espaços públicos, baseado no Plano de Ações Estratégicas para o Enfrentamento de DCNTs no eixo de promoção da saúde, com foco na atividade física.

A aplicação do questionário estruturado aos gestores municipais, conteve as seguintes variáveis: cargo que ocupa, formação, idade, tempo no cargo ocupado, sexo, presença ou ausência do Programa Intersetorial de Promoção da Saúde, presença ou ausência de parceria entre Secretaria de Saúde e Secretaria de Educação para promover atividade física e práticas corporais voltadas para crianças e jovens (jogos/atividades, avaliação do índice de massa corporal-IMC e outros índices de desenvolvimento educação em saúde), nas escolas municipais (mínimo duas aulas de educação física semanal, oferece atividade esportivas no contra turno e Programa Saúde na Escola) e espaços saudáveis (academia da saúde da terceira idade; com no mínimo um profissional de educação física por academia; capacitação para os profissionais que atuam na academia; academia da primeira idade; parques recreativos e ciclovias); quantidade de pista de caminhada/calçadas saudáveis, quadra poliesportivas e ginásio de esportes.

Em relação aos relatórios públicos do SISVAN, consultou-se os relatórios de crianças e adolescentes em fase escolar, na faixa etária de 5 a 19 anos, quanto ao estado nutricional: $\mathrm{SB}, \mathrm{OB}$ e $\mathrm{OB}$ grave. A coleta de dados ocorreu nos meses de abril a junho de 2014, sendo que os dados do SISVAN foram referentes ao período entre 2008 a 2013, já que foram os dados disponíveis na plataforma durante o período de coleta.

Tabulou-se os dados em planilhas, por meio do Programa Microsoft Excel, versão 2016. Inicialmente, realizou-se análise estatística descritiva simples dos dados coletados, os quais se apresentam em tabelas. As análises estatísticas se procederam por meio do software de Linguagem Estatística R, versão 3.5.1, através dos pacotes Tm e Qgraph.

Categorizou-se as ações desenvolvidas pelos municípios em três grupos de acordo com o percentual de ações implantadas em relação ao rol preconizado pelo PAEDCNT, sendo eles: Grupo I (GI) - composto por municípios que apresentaram 35\% a 50\%; Grupo II (GII) - composto por municípios onde este percentual variou de $51 \%$ a $65 \%$ e Grupo III (GIII) composto por municípios com 66\% a 80\% de ações implantadas ${ }^{8}$. Com base na caracterização de cada município participante do estudo confeccionou-se uma matriz de vizinhança com a co-ocorrência de ação entre os municípios. Essa matriz agregou a similaridade entre os municípios a partir da execução de ações semelhantes, sendo utilizada como suporte para a construção e análise de rede ${ }^{15}$.

Construiu-se a rede tomando cada município da matriz de vizinhança como um nodo, representação circular nas figuras. Esses nodos conectaram-se pelas hastes representando a intensidade da sua similaridade na matriz vizinhança (co-ocorrência de ações). Esses passos representam uma configuração de rede bipartite, na qual os municípios que executassem as mesmas ações teriam uma relação direta, já municípios que não executassem ações em comum, mas que tivessem relações diretas com um terceiro município apresentariam relações indiretas ${ }^{15}$.

Aplicou-se uma rede não direcional com algoritmo de posicionamento (Fruchterman-Reingold force-embedded), que permite aproximar nodos com co-ocorrência mais alta. Desta forma, quanto mais próximos os nodos (municípios) maior é a conexão entre os mesmos. As hastes apresentaram uma disposição pondera$\mathrm{da}$, de forma que a espessura exprime a força da haste que é a conexão entre dois itens. Estratificaram-se os nodos em escala de cinza, em função da prevalência de excesso de peso em crianças e adolescentes, dicotomizados a partir da média dos municípios ${ }^{16}$.

Customizou-se o tamanho dos nodos também em função do percentual de ações de promoção da saúde empregados por cada município, com nodos maiores representando maior percentual de ações. Deste modo, visualizou-se a concordância entre prevalência de excesso de peso (cores dos nodos) com o emprego de ações de promoção da saúde (tamanho dos nodos).

\section{Resultados}

Dos 30 municípios participantes, 56,7\% $(\mathrm{n}=17)$ possuíam apenas uma equipe de ESF, 23,3\% $(n=7)$ apresentavam entre três e oito equipes, $16,7 \%(\mathrm{n}=5)$ contavam com duas equipes e 3,3\% $(\mathrm{n}=1)$ dispunham de 66 equipes. Em relação ao total de Unidades Básicas de Saúde (UBS), 50\% ( $\mathrm{n}=15)$ dos municípios apresentavam uma unidade, $26,7 \%(\mathrm{n}=8)$ possuíam de cinco a onze unidades, $20 \%(n=6)$ possuíam duas e 3,3\% ( $n=$ 1) contava com uma rede formada por 29 UBS.

Das indicações dos gestores municipais para a entrevista, 27\% ( $=8)$ eram secretários de saúde e 73\% (n 
= 22) eram enfermeiros provenientes da ESF, da ABS, Coordenação da Saúde da Mulher e do Setor de Epidemiologia.

Quanto à idade, predominou a faixa etária entre 31 e 40 anos com 43\%, seguido de 20 a 30 anos com 33\%, 41 a 50 anos com 20\% e acima dos 50 anos com 3\%. Possuíam ensino superior completo ou maior titulação 80\%. Quanto ao tempo em que ocupavam o referido cargo ou função, $50 \%$ estavam na função há um período igual ou inferior a um ano, $26 \%$ exerciam a função entre 2 e $5,13 \%$ de 6 a $10,3 \%$ de 11 a 15 e $6 \%$ de 16 a 20.

Com base nos relatórios do SISVAN construiu-se um gráfico para analisar a evolução da prevalência de $\mathrm{SP} / \mathrm{OB}$ em crianças e adolescentes nos municípios do estudo. Verificou-se que no ano de 2008 as crianças e adolescentes apresentavam $31 \%(\mathrm{n}=37.588)$ e $24 \%$ $(\mathrm{n}=19.635)$ de SP e OB, respectivamente, e que este percentual cresceu gradativamente, alcançando 40\% (n $=46.878)$ e $43 \%(n=36.997)$ em 2013. Pode-se constatar ainda que, embora os adolescentes apresentassem uma prevalência de $\mathrm{SP} / \mathrm{OB}$ inicial menor em relação às crianças, apresentaram uma elevação maior destes índices no decorrer do período considerado e um percentual final também maior, como pode ser observado na Figura 1.

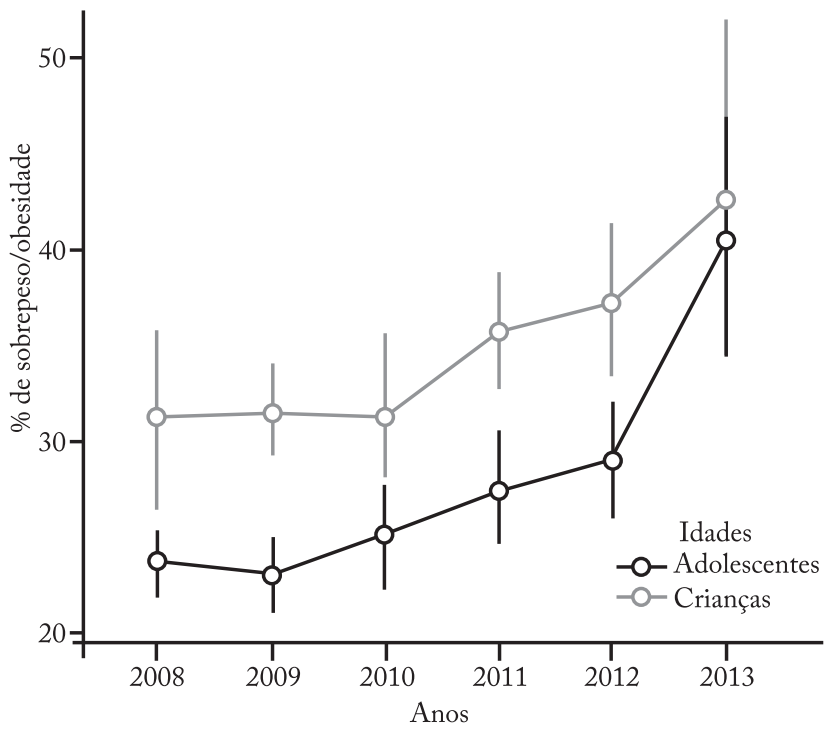

Figura 1 - Evolução do sobrepeso e obesidade em crianças residentes na 15ª Regional de Saúde do Estado do Paraná. Maringá, Paraná, 2019.

* $\mathrm{p}<0,05$

A quantidade de ações de PS implementadas pela totalidade dos municípios analisados e relacionadas à atividade física variou de $35 \%$ a $80 \%$, dos quais $56,7 \%$ $(\mathrm{n}=17)$ desenvolviam entre $35 \%$ a $50 \% ; 26,7 \%(\mathrm{n}=8)$ de 51 a $65 \%$ e $16,6 \%(n=5)$ desenvolviam ações entre 66 a $80 \%$ das preconizadas pelo PAEDCNT do MS. Destaca-se ainda que nenhum município desenvolvia mais que $80 \%$ das ações preconizadas.

Ressalta-se que o GI, que reuni os municípios com menor percentual de ações implantadas, apresentou também menor média de $\mathrm{SB}$ e $\mathrm{OB}$ em crianças e adolescentes. O GII, por sua vez, obteve a maior média da prevalência de SB em crianças, já o GIII revelou a maior média de prevalência em adolescentes. As adesões dos municípios às ações propostas pela PAEDCNT estão representadas na Tabela 1.

Tabela 1 - Adesão dos municípios às ações propostas pelo PAEDCNT. Maringá, Paraná, 2019.

\begin{tabular}{|c|c|c|}
\hline \multirow{2}{*}{ Ações indicadas pelo PAEDCNT } & \multicolumn{2}{|c|}{ Municípios } \\
\hline & $\mathrm{n}$ & $\%$ \\
\hline Programa Inter Setorial de Promoção da Saúde & 21 & 70,0 \\
\hline Parceria saúde/escola jogos/atividades & 6 & 20,0 \\
\hline $\begin{array}{l}\text { Parceria saúde/escola - avaliação de índice de massa corporal } \\
\text { e outros índices de desenvolvimento }\end{array}$ & 22 & 73,3 \\
\hline Parceria saúde/escola para educação em saúde & 27 & 90,0 \\
\hline Mínimo de duas aulas de educação física semanal & 13 & 43,3 \\
\hline Oferta de atividades esportivas no contra turno & 18 & 60,0 \\
\hline Adesão ao Programa Saúde na Escola & 22 & 73,3 \\
\hline Academias da Saúde & 29 & 96,7 \\
\hline Academia da Terceira Idade com no mínimo um educador físico & 6 & 20,0 \\
\hline $\begin{array}{l}\text { Capacitação profissional para os atuantes da Academia da } \\
\text { Terceira Idade }\end{array}$ & 2 & 6,7 \\
\hline Academia da primeira idade & 5 & 16,7 \\
\hline Parques recreativos & 17 & 56,7 \\
\hline Espaços saudáveis - ciclovias & 7 & 23,3 \\
\hline Pista de caminhada/calçadas & 18 & 60,0 \\
\hline Quadra poliesportivas & 26 & 86,7 \\
\hline Ginásio de esportes & 28 & 93,3 \\
\hline Feiras, eventos e outros programas saudáveis & 22 & 73,3 \\
\hline $\begin{array}{l}\text { Campanhas de comunicação e educação para prática } \\
\text { corporais/atividade físicas nos últimos seis meses }\end{array}$ & 12 & 40,0 \\
\hline $\begin{array}{l}\text { Estratégia de incentivo à prática de atividade física/práticas } \\
\text { corporais vinculadas à preparação para a Copa do Mundo e } \\
\text { Olimpíadas }\end{array}$ & 0 & - \\
\hline $\begin{array}{l}\text { Projeto de reformulação de espaços urbanos saudáveis em } \\
\text { andamento }\end{array}$ & 18 & 60,0 \\
\hline
\end{tabular}

PAEDCNT $=$ Plano de Ações Estratégicas para o Enfrentamento das Doenças Crônicas Não Transmissíveis; $\mathrm{n}$ = número total de municípios; $\%=$ porcentagem total de municípios.

Com base na construção de uma rede de similaridade das ações de PS desenvolvidas nos municípios, constatou-se que, tanto no grupo de municípios que apresentaram índices de excesso de peso em crianças 
ou em adolescentes abaixo da média quanto no grupo de municípios que apresentaram índices acima da média (Figura 2), a quantidade de ações de PS desenvol- vidas apresentou uma importante variação, de tal modo que ambos os grupos apresentaram municípios com poucas ou com várias ações de PS.

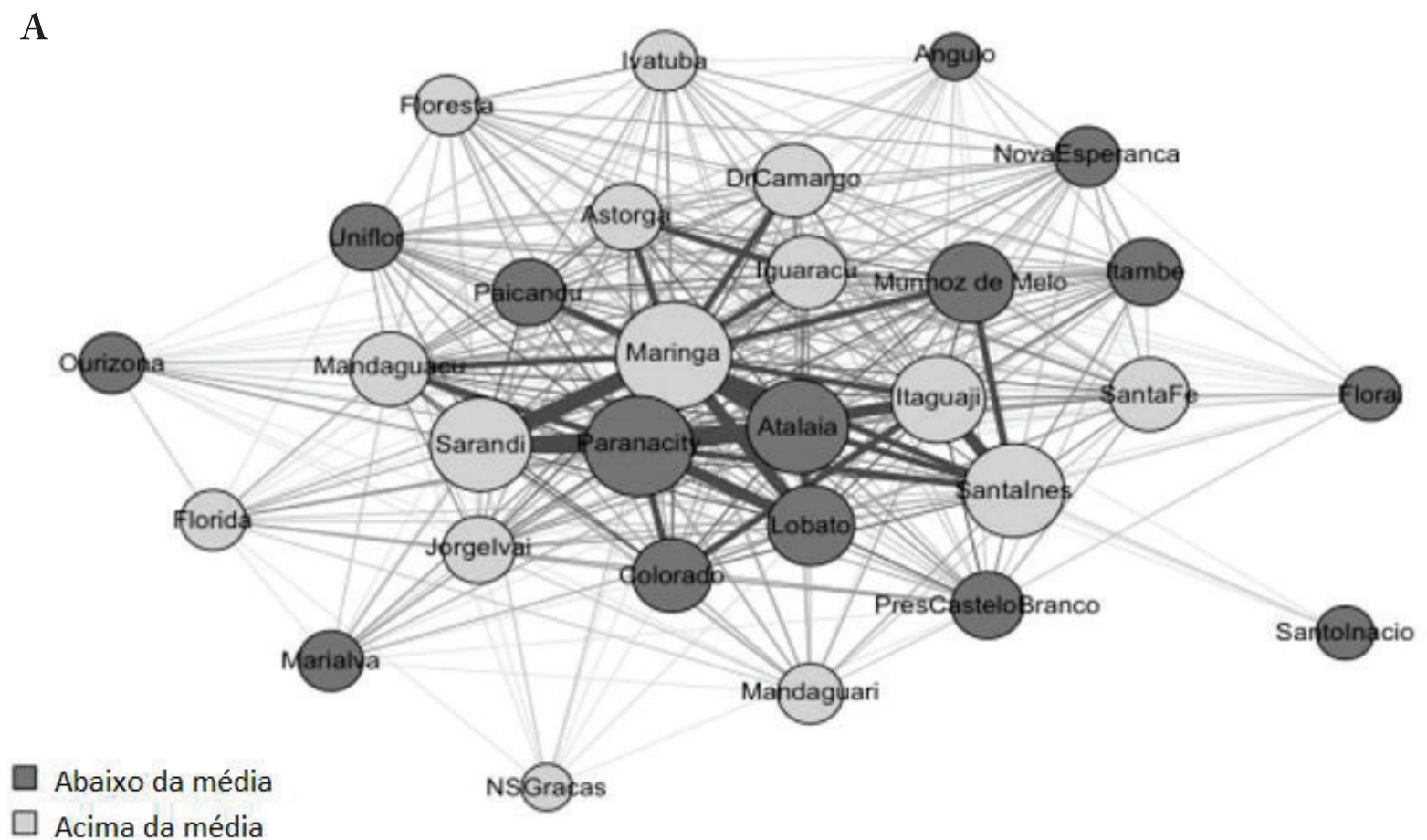

B

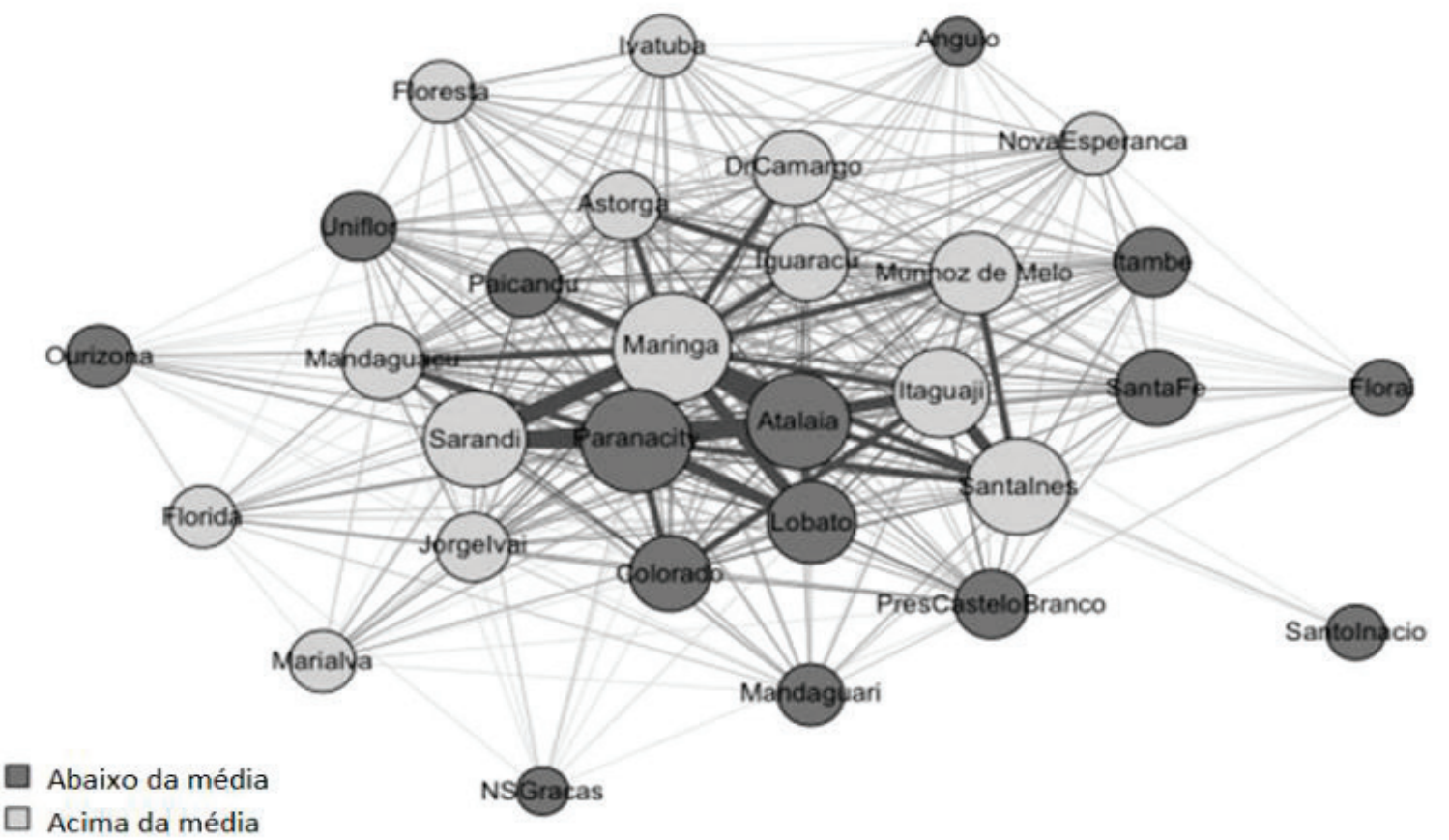

Figura 2 - Rede de similaridade entre Ações de PS realizadas nos municípios do estudo, estratificadas por prevalência de sobrepeso e obesidade em crianças e adolescentes. Maringá, Paraná, 2019.

As cores cinza claro e cinza escuro, representam a prevalência média de sobrepeso e obesidade. O tamanho dos nodos o percentual médio de ações desenvolvidas e a proximidade dos nodos e intensidade das hastes representam a associação entre as cidades. 
Ademais, evidenciou-se acentuada semelhança entre municípios de diferentes grupos, de tal forma que municípios com diferentes índices de excesso de peso em crianças e adolescentes demonstraram desenvolver ações similares de PS. A demonstração das redes de similaridades entre ações de PS realizadas entre os municípios conforme prevalência de $\mathrm{SB}$ e $\mathrm{OB}$ em crianças e adolescentes está na Figura 2 (A-Crianças; B-Adolescentes).

As cores cinza escuro e cinza claro, representam a prevalência de $\mathrm{SB}$ e $\mathrm{OB}$, o tamanho dos nodos o percentual de ações desenvolvidas e a proximidade dos nodos e intensidade das hastes representam a associação entre as cidades e traçou-se uma média considerando todos os municípios.

\section{Discussão}

No que concerne à distribuição de $\mathrm{SB}$ e $\mathrm{OB}$ em crianças e adolescentes encontrada a partir da consulta aos dados do SISVAN, verificou-se o crescimento acelerado nos índices deste agravo nos últimos 5 anos, aproximando-se ao índice nacional. Tais achados corroboram com a literatura encontrada, a qual aponta alta prevalência de excesso de peso e obesidade entre crianças e adolescentes em todas as regiões brasileiras ${ }^{17}$.

A tendência de evolução do agravo pode ocorrer devido eventuais falhas na alimentação dos dados na plataforma do SISVAN em seus anos iniciais de implementação, o qual aprimorou-se no decorrer dos anos, inviabilizando um diagnóstico fiel acerca do processo de evolução do SP/OB nestas faixas etárias ${ }^{18}$.

Com relação à análise da implantação de ações de PS voltadas à atividade física e controle do excesso de peso, observou-se que 56,7\% ( $\mathrm{n}=17)$ dos municípios desenvolvem até $50 \%$ das ações preconizadas. Tais resultados revelam a falta de priorização de investimentos nestas ações, contrariando o que seria esperado e necessário, especialmente devido aos crescentes índices de sedentarismo encontrados nesta população ${ }^{19}$.

Os dados da Pesquisa Nacional de Saúde do Escolar (PeNSE) revelam que apenas 30\% dos escolares são ativos, enquanto $63 \%$ encontram-se em situação insuficientemente ativa e quase $7 \%$ estão inativos. A PeNSE constatou ainda que o hábito de assistir a duas horas ou mais de televisão, em pelo menos um dia da semana, está presente em $78 \%$ dos estudantes investi$\operatorname{gados}^{8,20}$

Neste contexto evidencia-se a necessidade de trazer à pauta de discussões estratégias que busquem garantir estrutura para a prática regular de atividades físicas na infância e adolescência. Tais ações devem prever o acesso a estes benefícios por todas as classes sociais, garantindo a estrutura física e segurança que permitam a todos os cidadãos alternativas para o desenvolvimento de hábitos saudáveis ${ }^{21}$.

A literatura aponta que o acesso às áreas públicas de lazer interfere na prática de atividade física. Um estudo, realizado no Piauí, concluiu que a prática de atividade física de lazer variou significativamente conforme as condições socioeconômicas, onde adolescentes com melhor nível socioeconômico têm mais acesso a prática de atividades físicas ${ }^{22}$.

Observou-se ainda, na presente pesquisa, a ausência de Academias para a Primeira Idade (API), contrariando a necessidade imposta pelos indicadores atuais de $\mathrm{SB}$ e OB infantil ${ }^{8}$. Este achado pode denotar a falta de um olhar mais atento por parte das gestões municipais, no que tange à problemática crescente que este agravo vem representando para a sociedade ${ }^{23}$.

Outro fator a ser considerado que pode interferir na oferta de ações voltadas aos adolescentes é a dificuldade que a equipe da $\mathrm{ABS}$ apresenta para trabalhar com este público, o que conduz, invariavelmente, à proposição e desenvolvimento de ações esporádicas e pontuais e não de forma efetiva e contínua ${ }^{24}$.

No tocante às ações intersetoriais de PS, os resultados permitiram destacar as parcerias firmadas entre a Secretaria de Educação e a Secretaria de Saúde, visando ao desenvolvimento de ações de Educação em Saúde. Estes resultados refletem a visão dos profissionais de saúde, os quais percebem, no ambiente escolar, um espaço social que contribui para a promoção da saúde, já que é onde são formadas as opiniões dos educandos e de seus familiares ${ }^{25}$.

No entanto, as respostas dos gestores e seus indicados acerca da existência ou ausência das ações previstas do Programa Saúde na Escola, demonstraram que as intervenções desenvolvidas ainda se mostram muito pontuais, contrariando as recomendações expressas nos parâmetros curriculares nacionais, as quais estabelecem que os conteúdos de saúde devem estar inseridos no conteúdo curricular, por meio de abordagem transversal e interdisciplinar. Desta forma, a educação em saúde na escola, embora de extrema importância, tem sido pouco efetiva em sua prática, não conseguindo provocar mudanças de atitudes que conduzam a opções mais saudáveis de vida ${ }^{26}$.

Dentro da perspectiva de ampliação das ações de saúde no ambiente escolar, o Programa Saúde na Es- 
cola se firmou como ferramenta de ações intersetoriais, atingindo o objetivo de promover a saúde e a cultura da paz, reforçando a prevenção de agravos à saúde e fortalecendo a relação entre as redes públicas de saúde e de educação, proporcionando contato das equipes de ESF para avaliação das condições de saúde dos educandos ${ }^{20}$.

Ainda com relação às ações implementadas no âmbito do Programa Saúde na Escola, por meio das respostas dos gestores e seus indicados, detectou-se, baixo índice de atividades físicas, mesmo estas sendo consideradas um elemento importante para a formação educacional, podendo ainda auxiliar na detecção de fatores de risco à saúde levando a melhor qualidade de vida na fase adulta ${ }^{21,27}$.

Observou-se que a prevalência de $\mathrm{SB}$ e $\mathrm{OB}$ em crianças e adolescentes foi menor em municípios que possuíam baixo percentual de ações de PS implementados. Isto pode ser justificado pelo fato de que o Estado do Paraná ainda não implementou em todos os municípios as medidas de ações de prevenção e combate ao SB e $\mathrm{OB}$, como por exemplo, as academias de saúde que estão presentes em apenas $40 \%$ dos seus municípios ${ }^{28}$.

Verificou-se, através das respostas dos gestores e indicados uma grande variação de adesão a ações de PS nos municípios, o que permite refletir acerca das realidades locais, uma vez que municípios de diferentes tamanhos populacionais possuem condições estruturais, financeiras e de ordem profissionais diferentes, reforçando a necessidade de ações conjuntas intermunicipais, favorecendo as ações educativas de enfrentamento do $\mathrm{SB}$ e $\mathrm{OB}$ em crianças e adolescentes ${ }^{29,30}$.

As limitações do presente estudo encontram-se na manutenção inadequada de dados do SISVAN em seus primeiros anos ${ }^{30}$, seja pelo curto espaço de tempo transcorrido desde a implantação do PAEDCNT, inviabilizando uma avaliação completa de sua efetividade e de seus impactos. Outro fator limitante é a possibilidade das ações terem sido superestimadas, uma vez que os gestores não forneceram comprovações da implementação das ações, sendo necessário novos estudos sobre o tema no sentido de avaliar os efeitos destas proposições a médio e a longo prazo.

Dos trinta municípios analisados, nenhum apresentou mais de $80 \%$ de adesão de ações de promoção em saúde relacionadas às atividades físicas e práticas corporais para crianças e adolescentes, para o enfrentamento do SB e OB. No grupo de municípios com maiores taxas de adesão, encontram-se aqueles com maior número de população e de equipe de profissio- nais de saúde, evidenciando que as gestões municipais pouco têm avançado no sentido da criação de ambientes mais saudáveis e de estratégias de PS para crianças e adolescentes.

Faz-se necessário reforçar a interdisciplinaridade, reconhecendo os obstáculos estruturais, humanos e logísticos que ainda persistem no contexto da PS junto à clientela infanto-juvenil como pontos de partida para a busca de soluções a um estilo de vida mais saudável, com vistas à maior qualidade de vida futura. Portanto, sugere-se aos municípios o fortalecimento da parceria entre secretarias de educação e da saúde, com vistas ao planejamento de ações conjuntas adequadas para sua realidade local que auxiliem no enfrentamento do $\mathrm{SB}$ e $\mathrm{OB}$.

\section{Conflito de interesse}

Os autores declaram não haver conflito de interesses.

\section{Contribuição dos autores}

Victorino SVZ, participou da concepção inicial do estudo, redação e revisão crítica do texto. Shibukawa BMC, Rissi GP e Higarashi IH, participaram da análise dos dados, redação e revisão crítica do texto.

\section{Referências}

1. Paes ST, Marins JCB, Andreazzi A. Metabolic effects of exercise on childhood obesity: a current view. Rev Paul Pediatr. 2015;33(1):122-9.

2. Dias PC, Henriques P, Anjos LA, Burlandy L. Obesity and public policies: the Brazilian government's definitions and strategies. Cad Saúde Pública. 2017;33(7):e00006016.

3. World Health Organization [Internet]. Obesity and Overweight [citado 2019 Oct 12]. Disponível em: http:// www.who.int/en/news-room/fact-sheets/detail/obesity-andoverweight.

4. Jardim JB, Souza IL. Childhood obesity in Brazil: an integrative review. J Manag Prim Heal. 2017;8(1):66-90.

5. Crianças e Adolescentes em Dados e Estatísticas no Paraná [Internet]. Segurança alimentar e nutricional [citado 2020 Apr 28]. Disponível em: http://www.cadeparana.org.br/_ dimensoes/saude/seguranca-alimentar-e-nutricional/

6. Saraiva NCG, Medeiros CCM, Araújo TL. Serial album validation for promotion of infant body weight control. Rev. Latino-Am. Enfermagem. 2018;26:e2998.

7. Linhares FMM, Sousa KMO, Martins ENX, Barreto CCM. Childhood obesity: influence of parents on nutrition and lifestyle of children. Temas em Saúde. 2016;16(2):460-81.

8. Brasil. Ministério da Saúde. Plano de Ações Estratégicas para o Enfrentamento das Doenças Crônicas Não Transmissíveis no Brasil 2011-2022. Brasília, DF: O Ministério; 2011.

9. Brasil. Ministério da Saúde. Política Nacional de Promoção da Saúde: revisão da Portaria MS/GM no 687, de 30 de março de 2006. Brasília, DF: O Ministério; 2014.

10. Brasil. Ministério da Saúde. Secretaria de Atenção à Saúde. Informe situacional sobre os programas de alimentação e nutrição e de promoção da saúde na atenção básica, DF: O Ministério; 2017. 
11. Almeida LM, Campos KFC, Randow R, Guerra VA. Strategies and challenges of management of primary health care in control and prevention of obesity. Rev Eletr Gestão \& Saúde. 2017;8(1):114-39.

12. Conselho Nacional de Saúde. Resolução n. 466, de 12 de dezembro de 2012. Aprova diretrizes e normas regulamentadoras de pesquisas envolvendo seres humanos. Diário Oficial da União. 12 dez 2012; sec. 1, p. 59.

13. Conselho Nacional de Saúde. Resolução no 510, de 7 de Abril de 2016. Dispõe sobre as normas aplicáveis a pesquisas em Ciências Humanas e Sociais cujos procedimentos metodológicos envolvam a utilização de dados diretamente obtidos com os participantes ou de informações identificáveis ou que possam acarretar riscos maiores do que os existentes na vida cotidiana, na forma definida nesta Resolução. Diário Oficial da União. 24 Maio 2016; sec. 1, p. 46.

14. Instituto Brasileiro de Geografia e Estatística [Internet]. Sinopse do Censo Demográfico [citado 2020 Apr 30]. Disponível em: https://censo2010.ibge.gov.br/sinopse/

15. Newman MEJ. The structure and function of complex networks. SIAM Rev Soc Ind Appl Math Review. 2003;45(2):167-256.

16. Fruchterman TM, Reingold EM. Graph drawing by forcedirected placement. Software: Practice and experience. 1991;21(11):1129-64.

17. Fernandes BG, Meira JB, Souza P, Moreira LL, Guimarães PSS, Ferreira VA. Promoção de Saúde na Escola: estratégia para o controle do excesso de peso infantil. Rev Bras Obesidade, Nutrição e Emagrecimento. 2018;12(74):707-15.

18. Nascimento FA, Silva AS, Jaime PC. Coverage of assessment of nutritional status in the Brazilian Food and Nutritional Surveillance System, 2008-2013. Cad Saúde Pública. 2017;33(12):e00161516.

19. Reis AAC, Malta DC, Furtado LAC. Challenges for public policies aimed at adolescence and youth based on the National Scholar Health Survey (PeNSE). Ciênc Saúde Coletiva. 2018; 23(9): 2879-2890.

20. Brasil. Ministério da Educação. Pesquisa Nacional de Saúde do Escolar, DF: O Ministério; 2019.
21. Melo FT, Melo LT. Teaching strategy used in school physical education to prevent obesity in adolescent students. Rev Ciências \& Ideias. 2016;7(3):298-323.

22. Dumith SC, Santos MN, Teixeira LO, Cazeiro CC, Mazza SEI, Cesar JA. Practice of physical activity among youths in a city in the semiarid region of Brazil. Ciênc Saúde Colet. 2016;21(4):1083-93.

23. Becker LA, Rech CR, Hino AAF, Reis RS. Evidence-based decision making and promotion of physical activity among directors of local health departments. Rev Saude Publica. 2018;52:90.

24. Araújo MS, Sales LKO, Araújo MG, Morais IF, Morais FRR, Valença CN. Difficulties faced by nurses to develop direct actions to adolescents in primary care. Rev enferm UFPE on line. 2016;10(5):4219-25.

25. Souza KRS. Representações sociais de professores da educação profissional em saúde sobre o ser professor [dissertação de mestrado]. Natal: Universidade Federal do Rio Grande do Norte; 2018.

26. Neira MG, Borges CCO. Esquadrinhar e Governar: análise das recomendações do CONFEF para a Educação Física escolar. Educ Real. 2018;43(2):571-590.

27. Santos JPP, Prati ARC, Molena CAF. Association between blood pressure and indicators of obesity general and central in students: forms for care and education at children's in schools. Cienc Cuid Saude 2013;12(1):146-54.

28. Paraná. Plano de ações estratégicas para o enfrentamento das Doenças Crônicas Não Transmissíveis (DCNT) no Paraná 2012-2015. Curitiba, Paraná: Secretaria de Saúde; 2012.

29. Dias PC, Henriques P, Anjos LA, Burlandy L Obesity and public policies: the Brazilian government's definitions and strategies. Cad Saúde Pública. 2017;33(7):e00006016.

30. Ferreira J, Celuppi IC, Baseggio L, Savi D, Faganello VS, Souza JB. Regional planning of health services: what do the managers say? Saúde Soc. 2018; 27(1):69-79.

Recebido: 02/10/2019

Aprovado: $22 / 05 / 2020$

\section{Como citar este artigo:}

Victorino SVZ, Shibukawa BMC, Rissi GP, Higarashi IH. Promoção da Saúde: adesão municipal às açôes de combate ao sobrepeso e obesidade infanto-juvenil. Rev Bras Ati Fis Saúde.2019;24:e0102. DOI: 10.12820/rbafs.24e0102 